\title{
POWER DISAGGREGATION OF DOMESTIC SMART METER READINGS USING DYNAMIC TIME WARPING
}

\author{
Georgia Elafoudi, Lina Stankovic and Vladimir Stankovic
}

\author{
Dept. Electronic and Electrical Engineering, University of Strathclyde, Glasgow, UK
}

\begin{abstract}
Non-intrusive appliance load monitoring (NALM), also called load disaggregation, is a method for isolating the energy consumption of individual appliances from an overall household energy consumption. Traditionally, NALM techniques are based on measuring several electrical parameters at high sampling rates, which increases the meter cost and communications and storage overhead. In this paper, we propose a low-complexity disaggregation method based on Dynamic Time Warping algorithm that uses only active power aggregate smart meter data, captured at a low frequency, for training and disaggregation . Experimental results are provided for three households using data collected during two months, showing which individual appliances were used and when. Average recognition accuracy of $85 \%$ was obtained.
\end{abstract}

Index Terms - Smart Meter, NALM, Dynamic Time Warping, Disaggregation, Load Signature

\section{INTRODUCTION}

As the deployment of smart meters has already started, a lot of focus has been put on maximizing benefits of smart metering in terms of providing a simple pricing system and effective energy advice. To achieve this, it is not sufficient to have information about the energy consumption of the entire house, but it is necessary to break this consumption down to individual appliances. Non-Intrusive Appliance Load Monitoring (NALM), also referred to as NILM or NIALM, is a technique used to disaggregate household's power load without the need for individual appliance load sensors.

Load disaggregation is beneficial for all parties of the power network, as it can provide information about the status of an appliance (faulty or old), the changes in the load demand, even the pricing policy of energy providers. For this purpose, there is a number of research works that propose different NALM algorithms using a range of sampling rates (generally in the order of $\mathrm{kHz}$ ) and different electrical parameters for disaggregation. However, as commercial energy

This work is supported in part by the UK Engineering and Physical Sciences Research Council (EPSRC) project REFIT EP/K002708 and APAtSCHE EP/K002368, under the Transforming Energy Demand in Buildings through Digital Innovation (BuildTEDDI) funding programme. monitors provide sampling rates less than $1 \mathrm{~Hz}$, more focus is required for accurate and robust disaggregation techniques at low sampling rates.

In this paper, we propose a novel load disaggregation method validated using real data acquired using an energy wireless monitoring platform (EWMP) [1]. Active power data are acquired at sampling rates in the order of 1-10 seconds and sent to a dedicated remote server in the cloud in real time. The proposed disaggregation algorithm is of low complexity and is based on Dynamic Time Warping (DTW). The algorithm is tested using energy consumption data gathered for a period of two months from four different households following their everyday routines. The obtained results are competitive to those in the literature obtained at much higher sampling rates, demonstrating that DTW is a promising tool for power load disaggregation. This paper is organized as follows. Section 2 gives relevant background on NALM. Section 3 introduces the proposed machine learning disaggregation method, and Section 4 presents the experimental setup and performance analysis. Section 5 summarizes the key outcomes.

\section{BACKGROUND: NALM}

NALM, first proposed by Hart at [2] in the early 80 s, refers to disaggregation methods that aim to isolate the energy consumption of individual appliances from a total household energy consumption without the need of individual appliance sensors. The total power load is sampled at a sufficiently high resolution ([3], for example, samples at $1 \mathrm{~Hz}$ ), where each measurement is a vector of measured components, that describes the load. The two major load signatures that are used in literature are (i) one-dimensional load signature [4], comprising only the active power and (ii) two-dimensional load signature [5], comprising active and reactive power. Note, however, that other features can also be used for disaggregation, such as voltage and current waveform, electrical noise.

Many improvements of the original proposal of [3] have appeared recently. For example, in [6], Real-time Electrical Appliance Recognition (RECAP) system is based on an energy monitor attached to the main electrical unit, measuring active power, power factor, root mean square and peak current and voltage, and employing Artificial Neural Networks for 
classification. In [7], circuit-level energy measurements are used together with histogram thinning and Bayesian inference for recognizing small appliances, though the proposed method was tested using circuit-level measurements (with few test appliances) and it is not clear how it will perform in a home environment. In [8] a custom-made single point sensor was proposed for identifying the usage of resistive and inductive electrical load using transients; data were collected at $\leq 100 \mathrm{kHz}$ and $\leq 100 \mathrm{MHz}$ and standard machine learning techniques were used for events classification, with an overall accuracy of 85-90\%. Similarly, in [9], ElectriSense was used for identifying appliances that do not generate transients; classification was done using a KNN-based classifier and the obtained results show a mean identification and classification accuracy of $93.82 \%$. ViridiScope in [10] is a power monitoring system that uses existing infrastructure and sensors for sensing ambient signals from the appliances and a model-based machine learning algorithm is used for sensor calibration, and power consumption per-appliance was detected with an error of less than $10 \%$.

In [4] a factorial Hidden Markov model was used for disaggregation of active power load, with 1 minute sampling rate, using aggregate data and models of known appliances to create the signatures. The obtained accuracy is relatively low, i.e., accuracy of detecting microwave is $53 \%$ and tumble dryer is $69 \%$. In [11] appliance frequencies were used for appliance recognition and motifs based on a temporal ordering were extracted. Both research works used the REDD data set from [12], which has data sampled at 5 sec. Supero in [13] is a wireless sensor network, that combines light and acoustic information together with smart meter data and disaggregation using a K-mean unsupervised method for disaggregation. The above disaggregation methods were able to disaggregate appliance activity using low granularity, but they do not provide sufficient robustness.

Targeting at power load disaggregation using smart meters that are (or will be soon) present in average households (measuring active power only at sampling rates in the order or seconds or minutes), in the next section, we propose a lowcomplexity NALM algorithm that for the first time uses DTW for clustering.

\section{THE PROPOSED DTW-BASED DISAGGREGATION ALGORITHM}

The proposed disaggregation algorithm is a time-series based disaggregation approach that operates on windows of measurements obtained after edge detection and followed by clustering. It comprises a training and a testing phase.

During the training phase, aggregate active power data is used to create a library of appliance signatures (windows). Note that this phase is required whenever appliances change (i.e., addition, change or removal of appliances). The appliances are only classified using customers' daily diary or by cross-referencing the data with individual energy monitors if available. In the former approach, no two appliances should run in parallel during training.

To isolate appliance signatures, edge detection with adaptive thresholding is first performed. Only readings exceeding the threshold will be represented by a signature window. The threshold is automatically adapted depending on the minimum value present in the readings. High thresholds, on one hand, reduce the number of windows detected, avoiding small and unidentifiable windows, leaving only large power consuming events that can be more readily labeled as specific appliances, but on the other hand, remove possibility of detecting low-power devices, such as TV and lap-tops.

After edge detection, windows of events are created. A customised Dynamic Time Warping (DTW) method is then used instead of classic clustering, as in [3], for comparing and grouping windows from daily profiles and identifying unique load signatures. DTW is ideal for comparing vectors of different lengths, with non-identical values, and is a very popular tool in speech recognition (as seen in [14]) and lately in data mining. In the context of smart grids, it is used in [15] for clustering load profiles of electricity customers and in [16] a hybrid Hidden Markov Model and DTW algorithm were used in order to categorise residential water end use events. However, DTW has never been used before for NALM.

Given two windows of possibly different lengths, DTW performs a non-linear mapping of one window to another by minimizing the distance between them via dynamic programming. DTW is more effective in our problem than more conventional clustering, such as k-means or Density-based spatial clustering of applications with noise (DBSCAN), because the duration of appliance usage can significantly vary due to a change in appliance settings. Windows are matched to each other using DTW, removing a window when a match is already in the library. Therefore, a database of unique windows (class representatives) is formed.

The input to the testing phase is a time-limited reading, such as daily smart meter reading. After edge detection, each identified window is compared to each of the library windows using the DTW algorithm and labeled using the best match from the library. The output of the DTW classification is a soft value (minimum distance between the testing window and a window from the database). One example is shown in Figure 1, where solid (blue) line shows windows present in the library, while broken (red) line shows windows obtained in the test phase. We have a match if the accumulated distance [14] between the signature in the library and the one obtained during testing is below a threshold.

A correction step is finally used for separating appliances that might have not been distinguished correctly through the DTW algorithm. This step performs a check on the feature values at the instant before and after the detected appliance window. The distance between these values and the relevant start and end values of the window are compared and the min- 


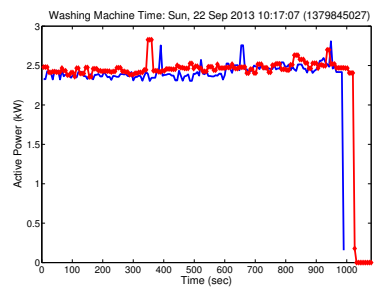

(a) Washing Machine

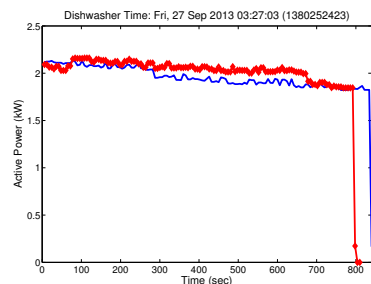

(b) Dishwasher

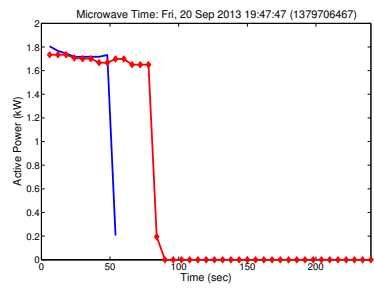

(c) Microwave

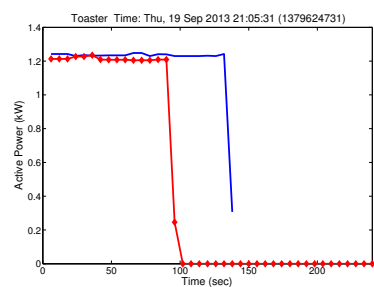

(d) Toaster

Fig. 1. Examples of appliance identification, database signatures (solid line) and detected signatures (marked line).

imum distance is compared to the thresholds defined for each used appliance. The final corrected identified appliances, together with the associated timestamp of the start of the event are the final output of the algorithm.

\section{EXPERIMENTAL SETUP AND ANALYSIS}

\subsection{Experimental setup and evaluation metrics}

We test and evaluate the proposed disaggregation algorithm using actual smart meter data from three households, which provides sampling rate of $6 \mathrm{sec}$. For the training phase, we use one week's worth of aggregate active power data, which does not contain overlapping or simultaneous operation with other appliances to build a library of signatures for expert classification. The validation and evaluation of the proposed algorithm is performed using the diary record kept by the residents, which were also used for classification during the training phase. The individual monitors were measuring active power from a wide range of typical household appliances, e.g., kettle, microwave, toaster, washing machine.

The evaluation metrics used are precision $(\mathrm{PR})$, recall (RE) and F-Measure (F-M) [17] defined as:

$$
\begin{gathered}
P R=T P /(T P+F P) \\
R E=T P /(T P+F N) \\
F M=2 *(P R * R E) /(P R+R E),
\end{gathered}
$$

where true positive (TP) presents the correct claim that the appliance was used, false positive (FP) represents an incorrect claim that an appliance was used, and false negative (FN) indicates that the appliance used was not identified.

\subsection{Performance Analysis}

The proposed method, as described in Section 3, requires an adaptive threshold in order to perform edge detection. The choice of threshold is governed by the desire to disaggregate high load appliances; we use a threshold of $500 \mathrm{~W}$. The algorithm provides as output the name of the appliance and the timestamp of the event and matches graphically the in-test operation with the library database. In Fig. 1, we provide a visual representation of some signatures, where the start time is also apparent.

Figs. 2 a, b and c represent the obtained accuracy per appliance and house for both precision, recall and F-measure. Note that the appliances presented with $0 \%$ accuracy, were either not available for testing in the specific household, or there was not enough information through the consumer's daily dairy for classification and evaluation of the specific appliances.

According to Fig.2c, dishwasher was recognized with high accuracy $90.91 \%$ in House 1 . Microwave was the appliance with the higher performance as for all houses the accuracy was more than $84.88 \%$. The obtained accuracy for House 1 and 3 for kettle was more than $93 \%$ and the recognition of electric shower was more than $87.38 \%$. Toaster was recognized with accuracy more than $75 \%$. Washing machine was identified with an accuracy of more than $71.42 \%$, as at some cases it was mismatched with dishwasher operations, as the washing cycle of both appliances is quite similar, see Fig.1a and 1b. This was also apparent for the dishwasher in House 2, where the dishwasher was identified with accuracy $79.15 \%$.

According to Table 1 the accuracy for all metrics is more than $82.18 \%$ averaged over the three houses, with the average F-M of over $85 \%$.

Table 1. Total Results
\begin{tabular}{|l|c|c|c|}
\hline & $P R(\%)$ & $R E(\%)$ & $F-M(\%)$ \\
\hline \hline House 1 & 99.11 & 83.37 & 90.56 \\
\hline House 2 & 89.38 & 82.18 & 85.63 \\
\hline House 3 & 100 & 91.04 & 95.31 \\
\hline
\end{tabular}

The resolution of the monitors used was $6 \mathrm{sec}$ which is relatively low compared to similar works as in [8]. Our proposed DTW-based algorithm was successful in recognising appliances with high consumption with accuracy over $85 \%$, which is a sufficient accuracy especially for aggregate data at low sampling rates [18] and is comparable with the $85-90 \%$ accuracy in [8] and the $90 \%$ in [10], where sampling rates were much higher and the energy sensors were bespoke. 


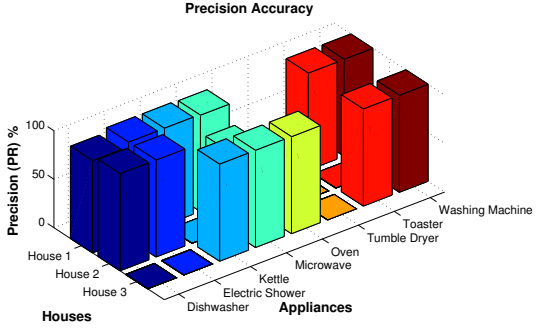

(a) Precision Accuracy (\%).

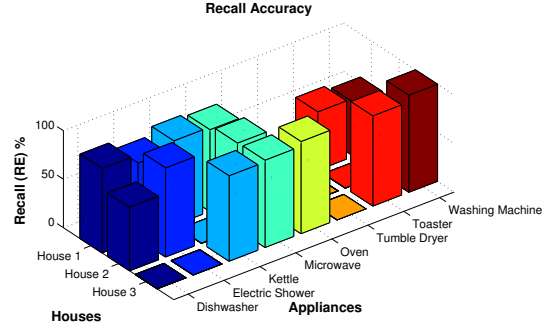

(b) Recall Accuracy (\%).

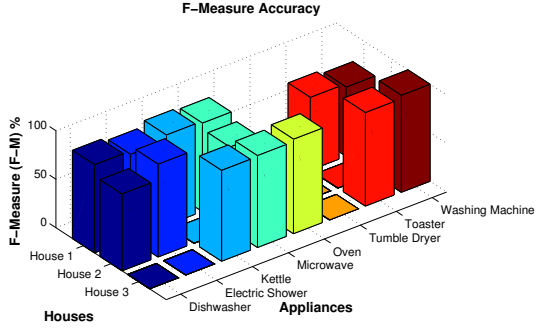

(c) F-Measure Accuracy (\%).

Fig. 2. Representation of the accuracy metrics per appliance for the tested households.

\section{CONCLUSION}

The paper proposed a solution for disaggregating real data using exclusively active power data for both training and testing at a low sampling rate of $6 \mathrm{sec}$. The proposed DTW-based algorithm was successful in recognising appliances with high consumption with an accuracy over $85 \%$ comparable to other proposed NALM algorithms in literature. Future work will focus on determining the limits in ability to detect devices, in order to isolate devices with similar active power and appliances with lower consumption, such as television.

\section{REFERENCES}

[1] A. Seeam, J. Liao, L. Stankovic, and V. Stankovic, "Improving energy efficiency with smart home appliance monitoring," in 7th Int. Conf. EEDAL, 2013.

[2] G.W. Hart, MIT. Energy Laboratory, and EPRI, Nonintrusive Appliance Load Data Acquisition Method: Progress Report, MIT Energy Laboratory, 1984.

[3] G.W. Hart, "Nonintrusive appliance load monitoring," Proc. IEEE, vol. 80, no. 12, pp. 1870-1891, 1992.

[4] O. Parson, S. Ghosh, M. Weal, and A. Rogers, "Nonintrusive load monitoring using prior models of general appliance types," in Proc. 26th Conf. Artificial Intelligence (AAAI-12), July 2012, pp. 356-362.

[5] A. Molina-Markham, P. Shenoy, K. Fu, E. Cecchet, and D. Irwin, "Private memoirs of a smart meter," in Proc. of 2nd ACM Workshop Embedded Sensing Sys. EnergyEfficiency in Building, NY, USA, 2010, BuildSys '10, pp. 61-66, ACM.

[6] A.G. Ruzzelli, C. Nicolas, A. Schoofs, and G.M.P. O'Hare, "Real-time recognition and profiling of appliances through a single electricity sensor," in 7th IEEE Int. Conf. SECON, 2010, pp. 1-9.

[7] A. Marchiori, D. Hakkarinen, Qi Han, and L. Earle, "Circuit-level load monitoring for household energy management," IEEE Pervasive Comput., vol. 10, no. 1, pp. 40-48, 2011.

[8] S.W Patel, T. Robertson, J.A. Kientz, M.S Reynolds, and G.D Abowd, "At the flick of a switch: Detecting and classifying unique electrical events on the residential power line," in Proc. Ubicomp, 2007, pp. 271-288.

[9] S. Gupta, M.S. Reynolds, and S.N. Patel, "Electrisense: Single-point sensing using emi for electrical event detection and classification in the home," in Proc. 12th Ubicomp, 2010, pp. 139-148.

[10] Y. Kim, T. Schmid, Z.M. Charbiwala, and M. B. Srivastava, "Viridiscope: Design and implementation of a fine grained power monitoring system for homes," in Proc. Ubicomp, 2009.

[11] H. Shao, M. Marwah, and N. Ramakrishnan, "A temporal motif mining approach to unsupervised energy disaggregation: Applications to residential and commercial buildings.," in AAAI. 2013, AAAI Press.

[12] J. Z. Kolter and M. J. Johnson, "REDD: A Public Data Set for Energy Disaggregation Research," in SustKDD Workshop Data Mining App. in Sustainability, San Diego, California, USA, 2011.

[13] D.E. Phillips, Rui Tan, M. Moazzami, Guoliang Xing, Jinzhu Chen, and D.K.Y. Yau, "Supero: A sensor system for unsupervised residential power usage monitoring," in IEEE Int. Conf. on PerCom, 2013, pp. 66-75.

[14] H. Sakoe and S. Chiba, "Dynamic programming algorithm optimization for spoken word recognition," IEEE Trans. Acoust., Speech, Signal Process, vol. 26, no. 1, pp. 43-49, 1978.

[15] F. Gullo, G. Ponti, A. Tagarelli, S. liritano, M. Ruffolo, and D. Labate, "Low-voltage electricity customer profiling based on load data clustering," in Procs IDEAS, NY, USA, 2009, IDEAS '09, pp. 330-333, ACM.

[16] K.A. Nguyen, R.A. Stewart, and H. Zhang, "An intelligent pattern recognition model to automate the categorisation of residential water end-use events," Environmental Modelling Software, vol. 47, pp. 108 - 127, 2013.

[17] D. L. Olson and D. Delen, Advanced Data Mining Techniques, Springer Publishing Company, Incorporated, 1st edition, 2008.

[18] A. Iwayemi, W. Wan, and C. Zhou, "Energy management for intelligent buildings," 2011. 